\title{
LETTER
}

\section{Between-centre differences for COVID-19 ICU mortality from early data in England}

\author{
Zhaozhi Qian ${ }^{1}$, Ahmed M. Alaa ${ }^{2}$, Mihaela van der Schaar ${ }^{1}$ and Ari Ercole ${ }^{3^{*}}$ (B)
}

(c) 2020 Springer-Verlag GmbH Germany, part of Springer Nature

\section{Dear Editor,}

Since the first cases in November 2019, the spread of SARS-CoV-2 infections has placed unprecedented strain on healthcare. The intensive care unit (ICU) is of particular concern as large numbers of patients with severe respiratory complications mean that in some areas, ICUs have been completely overwhelmed [1].

Understanding determinants of ICU outcome is crucial both for surge planning and shared decision making.
Whilst a number of risk scores have been published [2], they do not specifically look at this population. Furthermore, ICU availability, admission policy and structure vary across Europe [3] as do demographics and government policy. Thus, it is likely that ICU outcomes could also vary significantly by region motivating an individualised modelling approach. UK mortality has been particularly high, and we sought to urgently identify
A

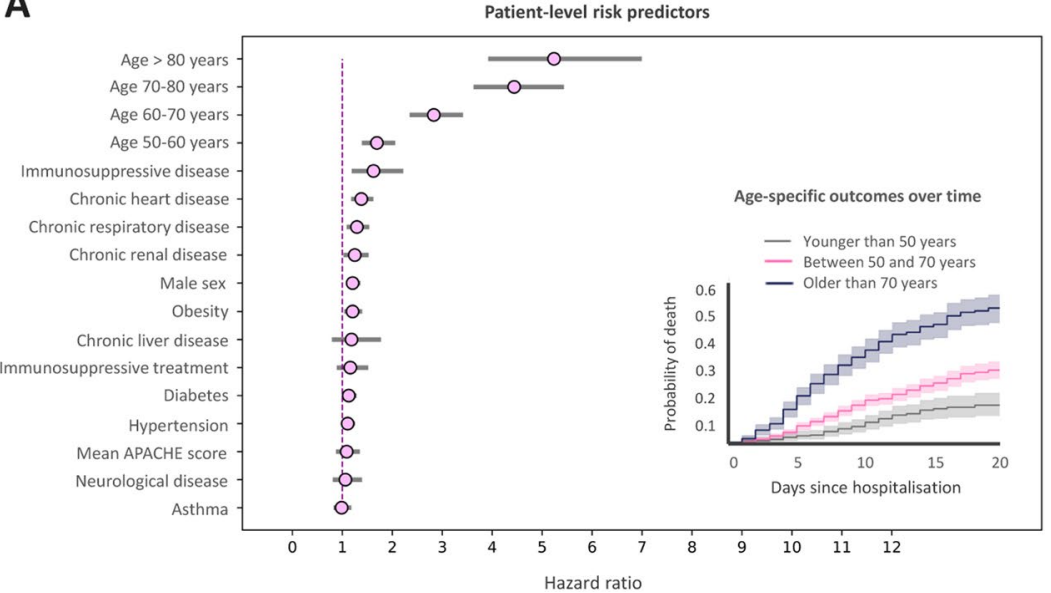

B

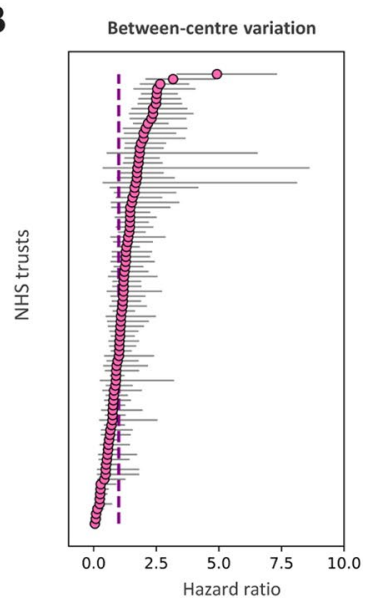

Fig. 1 a Fixed effects estimates for CHESS predictors and mean APACHE-II score for each site from national audit data. The strongest patient-factor predictors are older age, immunosuppressive disease and chronic heart/renal disease. The inset shows mortality probability over time for young, intermediate and older age groups. b Random effects showing between centres variation showing a hazard ratio variation between sites from 0 to over +4 which is comparable in magnitude to the strongest CHESS predictor (older age) showing that between-centres variation is an appreciable determinant of outcome. $95 \%$ Cls shown

*Correspondence: ae105@cam.ac.uk

${ }^{3}$ University of Cambridge Division of Anaesthesia, Addenbrooke's Hospital, Hills Road, Cambridge CB2 OQQ, UK

Full author information is available at the end of the article

\section{实 Springer}


predictors of mortality in patients admitted to the ICU with COVID-19.

We obtained de-identified COVID-19 Hospitalisation in England Surveillance System (CHESS) data from Public Health England (PHE) for the period from 8th February (data collection start) to 22nd May 2020 (5062 ICU cases-1547 deaths, 1618 discharges from 94 NHS trusts across England). Mean APACHE-II score for each site from ICU national audit data for COVID-19 patients over a similar period was used to attempt to correct for case-severity/as a proxy for admission policy since a variety of presentation severity indicators may also drive outcome [4].

We used a Cox proportional hazards mixed-effects model for mortality, with NHS trust as the random effect. The estimated coefficients for each predictor are shown in Fig. 1. The proportional hazards assumption was not violated ( $p=0.061$ ) (Supplementary material).

Immunosuppressive disease, chronic cardiorespiratory/renal disease and age were key predictors. Compared with these fixed effects, the magnitude of the between-centre variation (hazard ratio between 0 to over +4 ) is comparable to the strongest fixed effects predictor. The cause of such between-centre variation is unclear and may have a variety of residual case-mix or structural explanations. In particular, ICU demand varies both regionally and locally and we may hypothesize that high levels of strain or constraints on surge capacity could be actionable determinants, although we do not have data to examine this. Such considerations are important to understand as they may influence optimal configuration or transfer considerations locally.

Analysis limitations include possible incomplete ascertainment (particularly before $\sim 15$ th March), potential lead time bias from earlier deaths in the elderly group (although a sensitivity analysis excluding patients with $<7$ days follow-up yielded qualitatively similar results-Supplement Figure S1) and an inability to track patient transfers. Nevertheless, the magnitude of the random effects is striking. This motivates urgent comparative effectiveness research to characterise between-centre differences to inform surge best-practice in both in England and elsewhere.

\section{Electronic supplementary materia}

The online version of this article (https://doi.org/10.1007/s00134-020-06150-y) contains supplementary material, which is available to authorized users.

\begin{abstract}
Author details
${ }^{1}$ University of Cambridge Centre for Mathematical Sciences, Wilberforce Road, Cambridge CB3 OWA, UK. ${ }^{2}$ University of California, Los Angeles, Los Angeles, CA 90095, USA. ${ }^{3}$ University of Cambridge Division of Anaesthesia, Addenbrooke's Hospital, Hills Road, Cambridge CB2 OQQ, UK.
\end{abstract}

\section{Acknowledgements}

We would like to acknowledge PHE for providing us access to the CHESS dataset and the intensive care national audit and research centre for providing aggregate APACHE-II data, and also thank Anees Pari and Geraldine Linehan for their support.

\section{Author contributions}

AE and MvdS conceived of and supervised the study. ZQ and AMA coded the statistical models. All authors contributed to the manuscript.

Funding

Not externally funded.

Compliance with ethical standards

Conflicts of interest

The authors declare that they have no conflict of interest.

Availability of data and materials statement

Source data controlled by PHE. Under the terms of our data sharing agreement, the authors are not able to re-share the source data but will entertain requests for scientific collaboration.

\section{Publisher's Note}

Springer Nature remains neutral with regard to jurisdictional claims in published maps and institutional affiliations.

Accepted: 29 May 2020

Published online: 22 June 2020

\section{References}

1. Grasselli G, Zangrillo A, Zanella A, Antonelli M, Cabrini L, Castelli A, Cereda D, Coluccello A, Foti G, Fumagalli R, lotti G, Latronico N, Lorini L, Merler S, Natalini G, Piatti A, Ranieri MV, Scandroglio AM, Storti E, Cecconi M, Pesenti A (2020) Baseline Characteristics and Outcomes of 1591 Patients Infected With SARS-CoV-2 Admitted to ICUs of the Lombardy Region, Italy. JAMA. https://doi.org/10.1001/jama.2020.5394

2. Wynants L, Van Calster B, Bonten MMJ, Collins GS, Debray TPA, De Vos M, Haller MC, Heinze G, Moons KGM, Riley RD, Schuit E, Smits LJM, Snell KIE, Steyerberg EW, Wallisch C, van Smeden M (2020) Prediction models for diagnosis and prognosis of covid-19 infection: systematic review and critical appraisal. BMJ. https://doi.org/10.1136/Bmj.M1328

3. Rhodes A, Ferdinande P, Flaatten H, Guidet B, Metnitz PG, Moreno RP (2012) The variability of critical care bed numbers in Europe. Intensive Care Med 38:1647-1653

4. Wang K, Zhang Z, Yu M, Tao Y, Xie M (2020) 15-day mortality and associated risk factors for hospitalized patients with COVID-19 in Wuhan, China: an ambispective observational cohort study. Intensive Care Med. https:// doi.org/10.1007/s00134-020-06047-w 